\title{
A Complex Organic Modification on CNF Filler and Effects on Epoxy Resin Behavior
}

\author{
Yanling Bao ${ }^{1,} \mathrm{a}^{*}$, Hong Cui ${ }^{1}$, Xiaohu Zhang ${ }^{1}$, Baiya Wang ${ }^{1}$ and Xiuyun Wang ${ }^{1}$ \\ ${ }^{1}$ Xi'an Aerospace Composites Research Institute, 710025, P.R. China \\ abaojoya@qq.com
}

Keywords: Carbon Nano Fiber, Filler, Surface Modification, Epoxy Resin, Dispersion.

Abstract. Carbon nano fiber (CNF) was modified by acid oxidation and maleic anhydride (MA) graft in current modification. The CNF filler surface properties were characterized by energy dispersive spectrometer (EDS) and X-ray photoelectron spectroscopy (XPS). In addition, the properties of epoxy resin with modified CNF filler were indicated by thermogravimetric analysis (TGA) and mechanical analysis, and fracture morphologies were also observed by scanning electron microscope (SEM). The results show that the complex modification is a favorable surface modification for CNF filler, which would not only improve interfacial property between CNF and resin, but also increase the thermal decomposition temperature and mechanical character of epoxy resin.

\section{Introduction}

As a promising technology for composite, fillers have been successfully used for higher resin quality [1]. Carbon based nano fillers have outstanding specific surface area (SSA) and mechanical property [2], which have great effect on resin matrix [3]. During all kinds of carbon nano materials, CNF is one-dimensional material with diameter between carbon fiber and carbon nano tube, which not only have characters of CF (low density and high specific strength, etc.), but also possess advantages of nano materials (low defect and high SSA, etc.). Therefore, CNF is a promising filler to improve macroscopic properties of composite [4].

It's well known that pure nano carbon materials are all inert substance with high chemical and structure stability, so agglomeration is ubiquitous for Van der Waals force [5]. However, comprehensive properties of nano composites are well dependent on filler dispersion [6], and agglomeration of nano filler can become micron defect in matrix, which may lead to an invalid composite product. Therefore, it's necessary to produce functional grope on CNF filler by surface treatment for CNF dispersion in resin matrix.

In this paper, a complex modification composed of oxidation and MA graft was used on CNF. The results may be promising in preparing high-performance nano composites, and be helpful in studying dispersion mechanism of nano filler.

\section{Experiments}

Surface Modification and Characterization. CNF (Beijing DK nano technology Co. LTD) was chosen to be filler material and marked CNF-1. The modification of CNF was carried out as follows: CNF was added into concentrated nitric acid with ultraphonic osillation for $3 \mathrm{~h}$. Then oxidized CNF was reacted with MA in terms of graft in the solution of concentrated muriatic acid and ethyl acetate at $60^{\circ} \mathrm{C}$ for $6 \mathrm{~h}$ (marked CNF-2). CNF sample was washed and dried after treatments. Chemical property of CNF samples were observed by energy dispersive spectrometer (NORAN SYSTEM SIX, Thermo Electron Corporation, America) and X-ray photoelectron spectroscopy (Thermo Electron Corporation, America).

Resin Preparation and Analysis. The epoxy resin was composed of TDE-85 and E51, MOCA and DDM were used as curing agents. CNF-1 and CNF- 2 were respectively dispersed in TMPGE diluents 
agent before formula blending (the mass ratio of $\mathrm{CNF}$ and curing agents was $0.5 \%$ ). All used chemical reagents were analytically pure. Resin samples with CNF-1 and CNF-2 fillers were marked Sample-1 and Sample-2 respectively. Thermostability of Sample-1 and Sample-2 were represented by thermogravimemtric analyzer (TG209F3, NETZSCH Corporation, Germany), and mechanical properties were measured according to GB/T 2567-2008. Furthermore, fracture morphologies of samples were observed by scanning electron microscope (JSM-6460LV, JEOL Ltd., Japan).

\section{Results and Discussion}

Fig. 1 shows the EDS elemental analysis of CNF-1. As expected, Ni was the only impurity detected on $\mathrm{CNF}$ surface. The existence of $\mathrm{Ni}$ was unavoidable, because it's the catalyst for hydrocarbon dissociation during CNF preparation. Besides that, Fig. 1 also indicated that there was no organic functional group on CNF-1 surface.

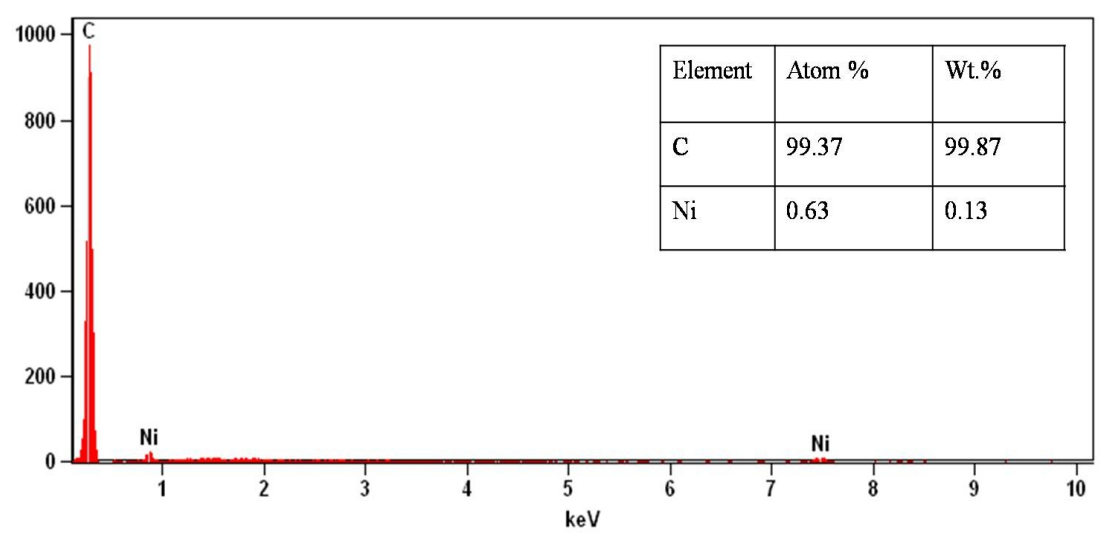

Fig.1 EDS of CNF-1 sample

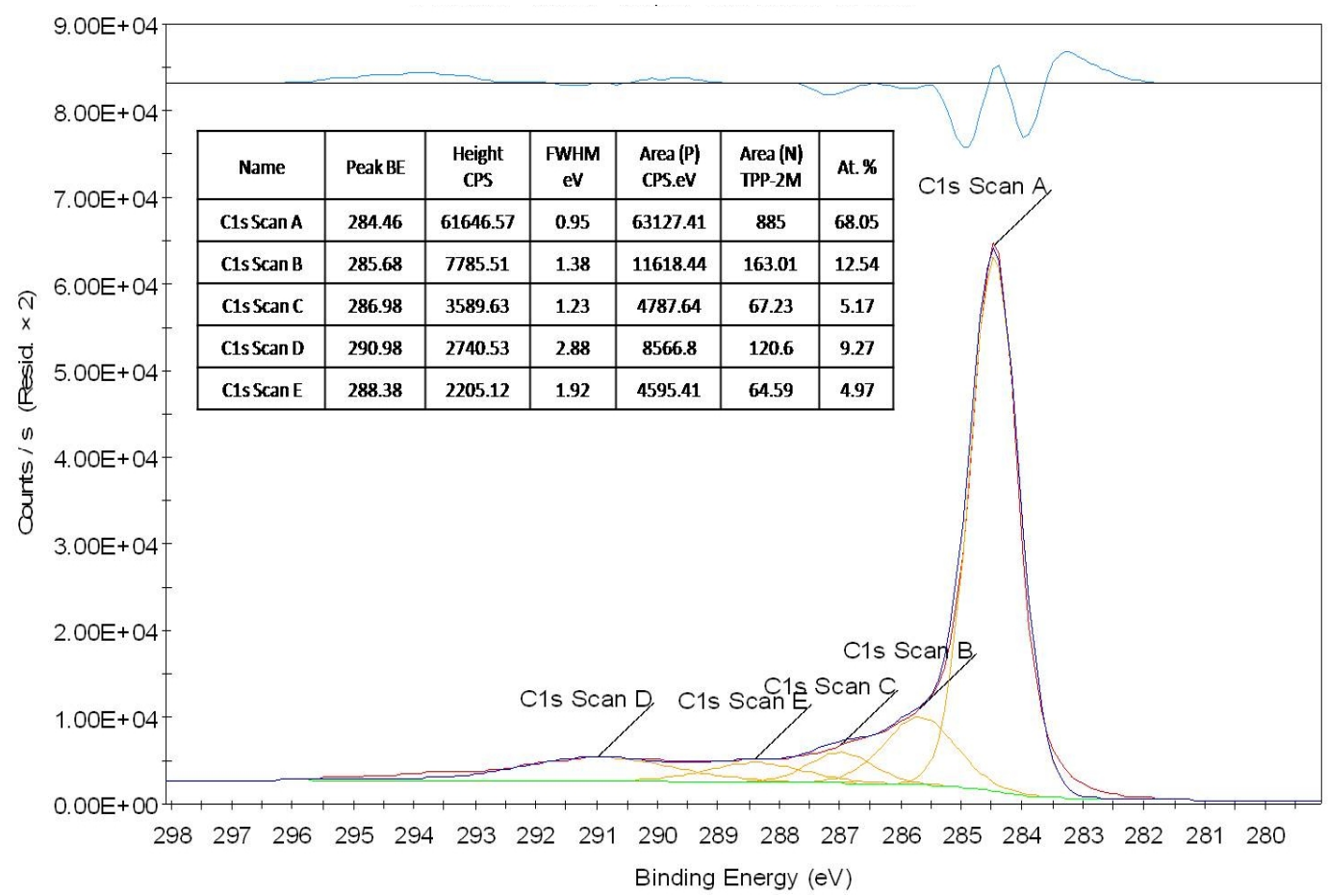

Fig. 2 C1s spectra of CNF-2

$\mathrm{X}$-ray photoelectron spectroscopy is a unique and valid technique for obtaining detailed information about the atomic structures of elements and molecules from carbon surface. The $\mathrm{C} 1 \mathrm{~s}$ core region spectra from XPS of CNF-2 is revealed in Fig. 2 and used to express concentration of oxygen groups by computing areas of the peaks in C1s spectra. Peak A, B, C, D and E in Fig. 2 represent C-C, C-O, 
$\mathrm{C}=\mathrm{O}, \mathrm{CO}_{3}{ }^{-2}$ and $\mathrm{O}-\mathrm{C}=\mathrm{O}$ respectively, and the mainly functional groups on $\mathrm{CNF}-2$ are $\mathrm{C}-\mathrm{O}, \mathrm{C}=\mathrm{O}$ and $\mathrm{O}-\mathrm{C}=\mathrm{O}$. Besides that, the content of total oxygen group reaches to $31.95 \%$, which indicates $\mathrm{HNO}_{3}$ oxidation and MA graft are effective surface modifications to produce organic groups on CNF.
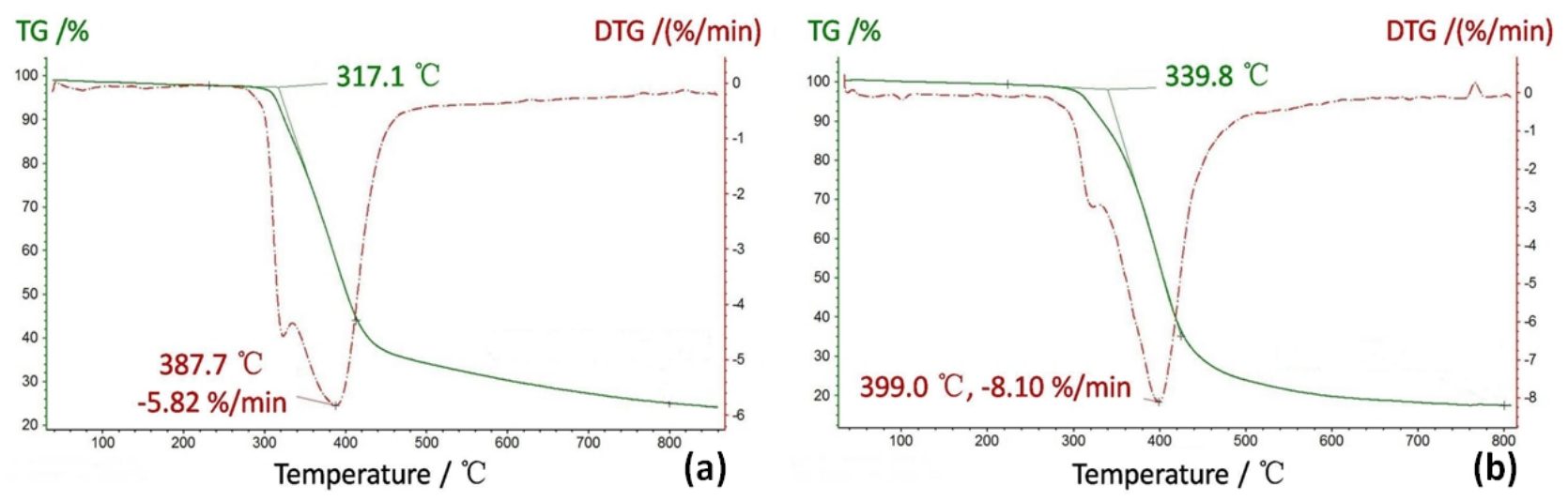

Fig. 3 TG curves of Sample-1 (a) and Sample-2 (b)

Fig. 3 illustrates the TG curves of epoxy resin with different CNF fillers after thermocuring. While temperature gradually increasing, Sample-1 with CNF-1 filler started to decompose at $317.1^{\circ} \mathrm{C}$. However, the starting decomposition temperatures of Sample- 2 with CNF-2 filler reached to $339.8^{\circ} \mathrm{C}$, which is much higher than that of Sample-1.

Compared with normal polymer, carbon kind nano materials have much higher decomposition temperature, and CNF filler can inhibit movement of molecular chains when composite is heated, which will theoretically result in better thermostability of the whole composite system. However, this would only happen if CNF filler has been well distributed in matrix and produced favorable network structure, so that heat can conduct promptly and uniformly in composite, which will restrain decomposition caused by heat accumulation. Fig. 3 implies the oxidation and graft surface treatments on CNF filler contributes greatly to improve sample thermostability, which means organic groups produced by the complex modification have successfully optimized dispersibility of CNF in epoxy resin.

Table 1 Mechanical property of different samples

\begin{tabular}{ccccc}
\hline Samples & $\begin{array}{c}\text { Tensile strength } \\
(\mathrm{MPa})\end{array}$ & $\begin{array}{c}\text { Tensile modulus } \\
(\mathrm{GPa})\end{array}$ & $\begin{array}{c}\text { Compressive strength } \\
(\mathrm{MPa})\end{array}$ & $\begin{array}{c}\text { Compressive modulus } \\
(\mathrm{GPa})\end{array}$ \\
\hline Sample-1 & 90.2 & 3.64 & 154 & 3.40 \\
Sample-2 & 110 & 3.98 & 168 & 3.37 \\
\hline
\end{tabular}

Mechanical property data of composite samples with different CNF filler are shown in Table 1. It's obvious that tensile and compressive performance of Sample-2 were better than that of Sample-1, which indicates the complex modification of CNF filler can actively affect mechanical property of composite. In addition, Fig. 4 demonstrates the fracture morphologies of Sample-1 and Sample-2. It's clear that fracture of Sample-1 is brittle rupture with river pattern, and most of CNF-1 filler has been pulled out after fracture, which demonstrates the interface property between CNF-1 and matrix is terrible. However, Sample-2 exhibits ductile fracture with dimples and no CNF-2 has been found after rupture, which means organic groups produced by complex modification on CNF have availably improved the ductility of composite and the interface property between CNF and matrix. 


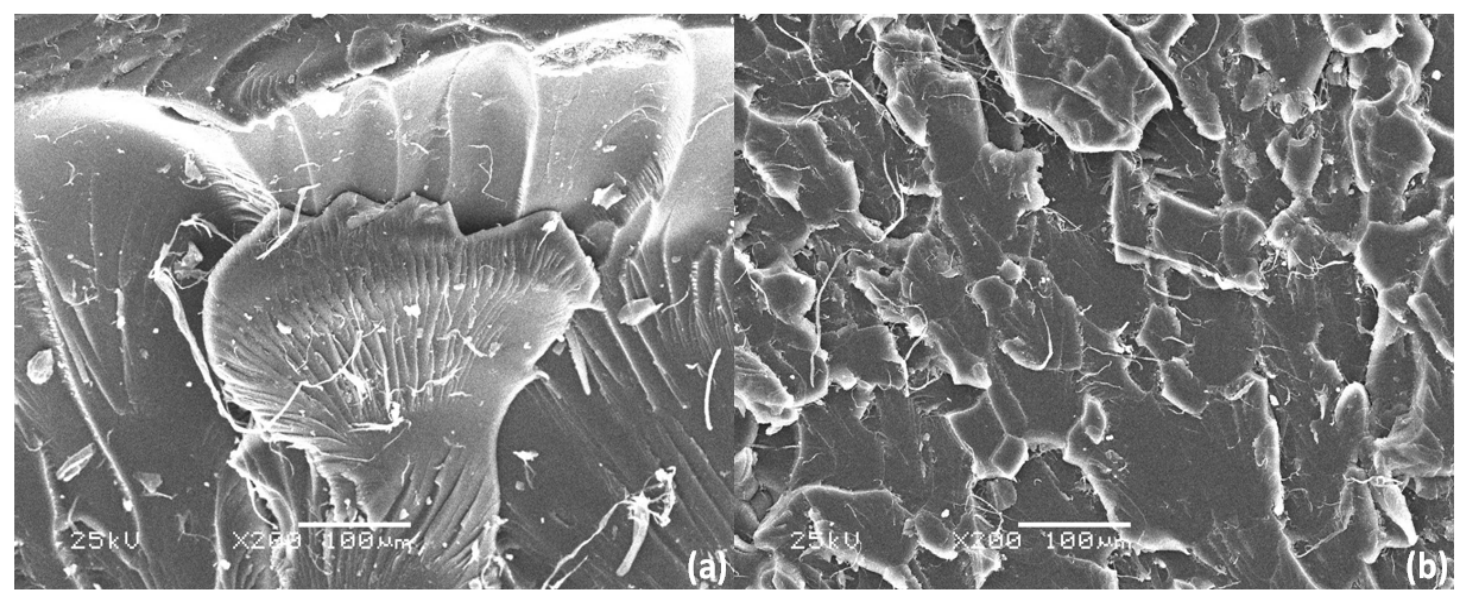

Fig. 4 Fracture morphologies of Sample-1 (a) and Sample-2 (b)

\section{Summary}

The complex modification on CNF composed of surface oxidation and MA graft is regarded to be a favorable surface treatment for CNF filler in nano composite. The organic groups produced by the complex modification optimized dispersibility of CNF in epoxy resin, which could contribute greatly to improve sample thermostability. Furthermore, the complex modification on CNF filler can actively affect mechanical property of composite, and availably improve the interface property between CNF and matrix.

\section{References}

[1] M. Hussain, et al. Mechanical property improvement of carbon fiber reinforced epoxy composites by $\mathrm{Al}_{2} \mathrm{O}_{3}$ filler dispersion [J]. Materials Letters, 1996, 26(3): 185-191.

[2] Y. Zhu, et al. Graphene and Graphene oxide: synthesis, properties, and applications [J]. Advanced Material, 2010, 22(35): 3069-3024.

[3] H. Jang, et al. Interfacial shear strength of reduced grapheme oxide polymer composites [J]. Carbon, 2014, 77: 390-397.

[4] S. Kumar, et al. Fibers from polypropylene/nano carbon fiber composites [J]. Polymer, 2002, 43(5): 1701-1703.

[5] Y. Si, et al. Synthesis of water soluble graphene [J]. Nano Letters, 2008, 8(6):1679-1682.

[6] H. Kim, et al. Graphene/polymer nanocomposites [J]. Macromolecules, 2010, 43(16) : 6515-6530. 